\title{
Development of 3D Visibility Analysis Models Using NURBS in A Residential Development
}

\author{
ChoongSik Kim ${ }^{1}$, Hoon $\mathrm{Ko}^{2}$ and KyungJin $\mathrm{An}^{3}$ \\ ${ }^{1}$ Dept. of Traditional Landscape Architecture, \\ Korea National University of Cultural Heritage, 323-812, Buyeo-gun, Korea \\ ${ }^{2}$ The Department of Informatics, J. E. Purkinje University, \\ J. E. Purkinje University Faculty of Science, Ceske mladeze 8, \\ 400 96, Usti nad Labem, Czech Republic, \\ ${ }^{3}$ Department of Environmental Planning, Konkuk University \\ 120 Neungdong-ro, Gwangjin-gu, Seoul, 143-701 South Korea \\ ${ }^{1}$ kimch@nuch.ac.kr, ${ }^{2}$ hoon.ko@ujep.cz, ${ }^{3}$ dorian@konkuk.ac.kr
}

\begin{abstract}
Within planning process, landscape visibility assessment has advantages of identifying landscape character and changes. As a planning tool, planners and other professionals are able to exam various planning scenarios effectively. However, current visibility analysis maps have been produced under raster driven information, which lacks in detail and its accuracy. In response of current phenomena of production of landscape visibility analysis and adaptation within planning process, the research aimed to develop an advanced visibility analysis programme based on vector data (NURBS) in order to improve accuracy of the process and result. In order to rectify of the new application, a residential development in Seoul has been chosen as case study. With newly produced visibility analysis produced from $V E 3 D$, the study analyses terrain, high-rise building blocks and any built up structures in $3 D$ forms. After case study, VE3D which was developed for visibility analysis from vector-based datasets was able to illustrate high level of detail in visibility identification better than using conventional Arcview 3.3 programme. Moreover, VE3D can produce a visibility analysis in reflecting complicated structures such as flyovers and high-rise tower blocks. The research also suggests that VE3D is able to contribute to in-depth and credible visibility analysis and this can be achieved by further research on application within various terrains and built up structures.
\end{abstract}

Keywords: VE3D, Planning Aid, Landscape Visibility, Computer Visualisation

\section{Introduction}

Implementing Geographic Information System (GIS) applications is known to an effective way of evaluating visibility for wider area in decision-making process within development management. In particular, using grid based spatial data is highly effective to generate analysis plans and maps. The process of Landscape Visibility Analysis enables planners to identify potential landscape character and its changes. In particular, the visibility analysis based on GIS application can be efficient, intuitive and economic [1][2]. Furthermore, Cumulative Visibility and Exposure Analysis provide extensive analysis outcomes, which benefits from GIS applications [2]. However, there is a danger that the GIS based analysis could be biased depending on height of grid cell size; moreover, each grid cell contains only 
single height information (value) which cannot handle irregular built up structures and floating bridges. On the other hand, commercial software such as Ecotect or Geoweb 3D can be used for a vector data-based visibility analysis in order to overcome such raster based lattice structure model [3]. These vector-based models can simulate bridges, flyover, built ups, terrain information as close to the real world. Even though such software adopts raytracing methods to increase realism and efficiency, there is no single tool for visibility analysis yet [2].

Therefore, this research aims to develop a vector data driven 3D visibility analysis programme in order to increase accuracy of the analysis [3-5]. Integrating NURBS data new 3D visibility analysis software would be designed to handle terrain data efficiently. As well as developing a vector data driven 3D visibility analysis application, the study employs a case study of housing development in order to prove the effectiveness and efficiency of the 3D Visibility Analysis process [6, 7]. A residential development in the city of Seoul will adopts this analysis process for its siting and visual impact assessment. Through the case study analysis, the study aims to provide credibility of the newly developed process and seek its adaptation within planning process. This research is divided into five sections. Section 1 introduces the background, research questions, and overall organisation of the study. Section 2 attempts to conceptualise visibility analysis in planning. It also reviews historical backgrounds and definitions to reach the most appropriate concept of the research. Thereafter, it presents the main points of visibility analysis process together with emerging issues of current use of the technology in planning through literature review. Section 3, methodology is divided into two sections with each addressing site selection/viewpoint and research methodology/process. Section 4 deals with findings and results. Finally, section 5 concludes the study with recommendations.

\section{Process of Visibility Analysis}

Raster driven visibility analyses employ ground models based on the datasets such as Triangular Irregular Network (TIN), Stepped Regular Square Grid (sRSG), Triangulated (tRSG). In particular, sRSG datasets are widely used because its faster data processing speeds as well as enabling simulations with smaller sizes of datasets [Juan M, 11][ J.B, 11]. Visibility Analysis based on sRSG data models draws visible sprays from viewpoints to targets, then analyses and calculates any obstruction within the sprays. Grid methods, however, calculate from ground models to built-up structures; therefore, it is difficult to analyse high rise tower blocks or flyovers. While vector data driven Visibility Analyses principally calculates between viewpoint and target points and renders results based on the sprays, the Visibility Analyses used mesh and surface have benefits of accurate form making and efficiency on realistic modelling. However, in order to increase the level of detail in terrain, significant numbers of meshes are needed then, consequently increase computing time. Moreover, computing can be bottlenecked when it calculates the visual sprays [6][9]. Conversely, NURBS models use splines and they can express complicated terrains quickly with relatively small number of nodes. Moreover, NURBS can provide tools such as rendering, shades, visibility, and transformation for terrain analysis. Models produced by NURBS curves can be used for Visibility Analysis with ray tracing and division methods; therefore, computing process is very fast and deal with terrains in relatively small file sizes [10].

Producing Visibility Analysis is divided into two depending on number of viewpoints; view shed and cumulative view shed. Cumulative view shed is adding results of more than one singular viewpoint and normally used for multiple viewpoint analysis in highly sensitive 
areas. However, it has been limited for reflecting visual perceptions and visibility distances since it simply accumulates visibility values of single viewpoints using bit data $(0$ and 2 datasets). Therefore, Cumulative Visibility Analysis which takes human perception into accounts has been required [10]. Vector methods can compute visible distance from targets; whereas, raster based methods only produce Visibility Analysis with simple accumulation bits by bits.

\section{Case Study - A Residential Development in Seoul}

Seoul Forest Apartment and Kum-Ho 14 Redevelopment District have been chosen as case study and site in order to review terrain, high-rise building blocks and any built up structures at the same time. Because the site is located in the area where green open space is adjacent and within a high dense high rise apartment blocks as well as there is Du Mo Bridge, which is a concrete built flyover within walking distance [11]. This enables us to review various structures and terrain. Total 15 viewpoints were selected on the basis of visibility mainly from (1) footpath towards Ung-Bong Mountain, Du Mo Bridge and (3) Han River Ferry routes.

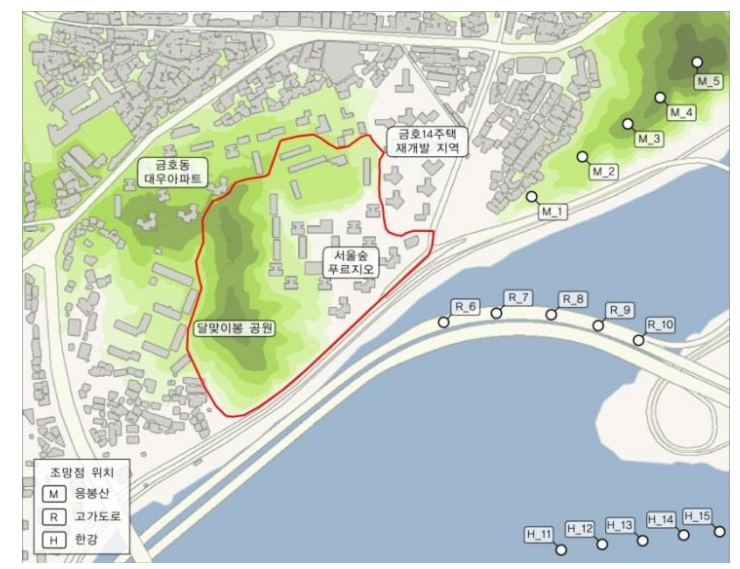

Figure 1. Case Study Site and Viewpoints

Database was constructed by both rasterised and vector data driven software. Then, Visibility and Cumulative Visibility Analysis have been carried out. In case of Rasterised datasets, Arcview 3.3 was employed to assemble grids from DTM 1:1000 and the analysis was carried out from the grids.

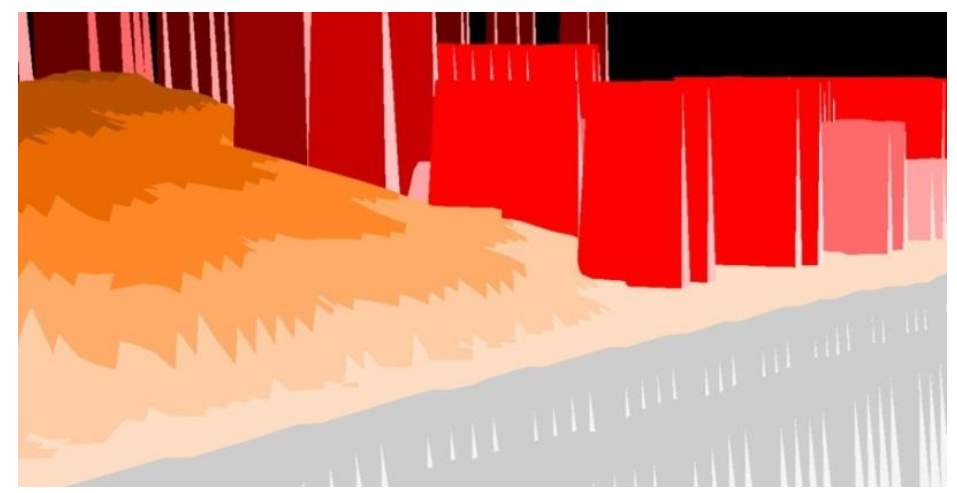

Figure 2. 3D Terrain and Buildings Used in Visibility Analysis 1 (from ArcView Elevation) 
Whereas, for vector based datasets, Rhinoceros 5.0 was implemented for authorising NURBS surfaces for the terrain of the area, then other built up structures such as apartment blocks and concrete flyover were also converted as surface in order to combine with the terrain. VE3D module were utilised for analysing the datasets.

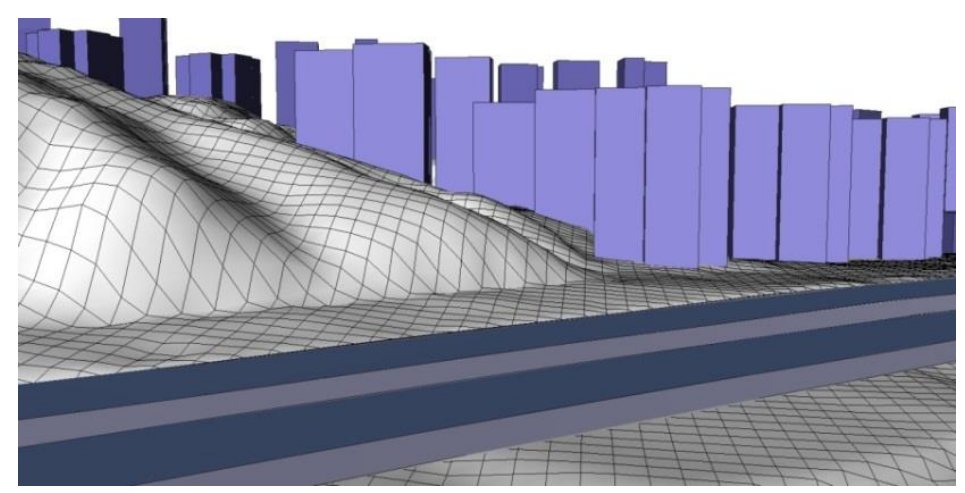

Figure 3. 3D Terrain and Buildings Used in Visibility Analysis 2 (from
Rhinoceros - NURBS) Rhinoceros - NURBS)

For the both datasets, $1 \times 1,5 \times 5$, and $10 \times 10 \mathrm{~m}$ grids terrains were compared allowing differences in level of detail. However, in the grid 1x1, VE3D could not handle the amount of time for analysis and consequently excluded in the research.

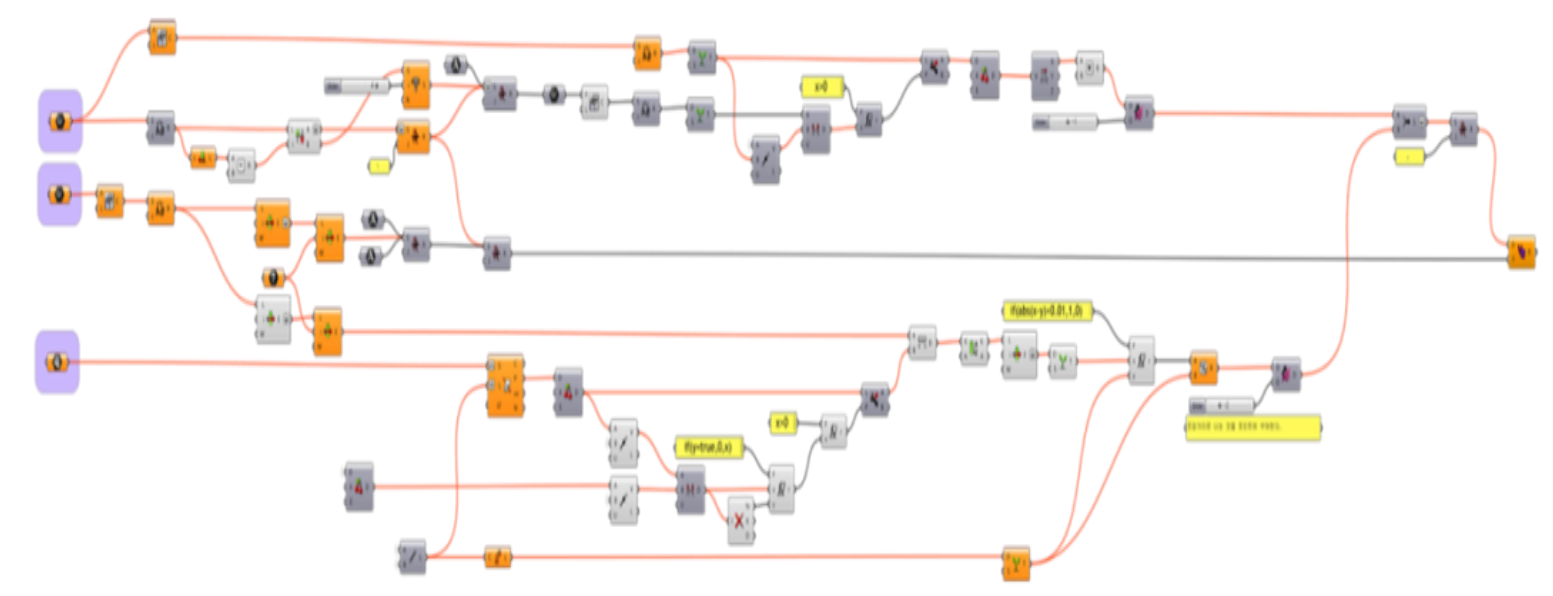

Figure 4. Visibility Analysis Process by VE3D

The both analyses were carried out using same hardware; Intel Core i5 $7602.80 \mathrm{GHz}$ CPU, 4GB DDR3 RAM, NVIDIA GeForce GTS 250, 7200 RPM SATA HDD and Windows 7 operating system.

\section{Result \& Findings}

\subsection{Visibility Analysis Processing Time-VE3D}

Before the actual construction start, Visibility Analysis processing time by VE3D for $5 \times 5$ grids is minimum 10 minutes 26 seconds (viewpoint 3, 4) and maximum 13 minutes 24 seconds (viewpoint 7), which the average time become 11 minutes 8 seconds. For 10x10 
grids, processing time is minimum 2 minutes 49 seconds (viewpoint 3) and maximum 3 minutes 3 seconds (viewpoint 15), which the average time become 2 minutes 54 seconds. There is an increase for analysis after completion. For instance, for $5 \times 5$ grids Visibility Analysis processing time was minimum 13 minutes 27 seconds (viewpoint 10) and maximum 25 minutes 33 seconds (viewpoint 7), which the average time become 18 minutes 1 second. For 10x10 grids, processing time is minimum 3 minutes 5 seconds (viewpoint 13) and maximum 5 minutes 37 seconds (viewpoint 14), which the average time become 3 minutes 52 seconds. This incensement could be explained by the fact that there were increase number of built up structures such as 30 tower blocks in after completion, the process time for this blocks' concealment and openness increased the processing time by approximately $1.5-2$ times. We can therefore, conclude that multiple viewpoint Visibility Analysis for 5x5m grids models is less efficient since it processes longer than 30 minutes. However, for 10x10m grids models process within around 3 minutes and therefore, they have potential to use for multiple Visibility Analysis.

Table 1. VE3D's Visibility Analysis Processing Time (unit - min:sec)

\begin{tabular}{|c|c|c|c|c|c|c|c|c|c|c|c|c|c|c|c|c|c|}
\hline \multirow{2}{*}{$\begin{array}{l}\text { View } \\
\text { change }\end{array}$} & \multirow{2}{*}{ Grids } & \multicolumn{15}{|c|}{ Viewpoint } & \multirow{2}{*}{ Ave. } \\
\hline & & 1 & 2 & 3 & 4 & 5 & 6 & 7 & 8 & 9 & 10 & 11 & 12 & 13 & 14 & 15 & \\
\hline \multirow{2}{*}{ Before } & $5 \times 5 \mathrm{~m}$ & 10:41 & 11:24 & 10:26 & $10: 261$ & $10: 29$ & $\mid 11: 29$ & $13: 24$ & $10: 511$ & $10: 51$ & $10: 51$ & $11: 15$ & 11:04 & 411:00 & $\mid 11: 14$ & $11: 42$ & 11:08 \\
\hline & $10 \times 10 \mathrm{~m}$ & $02: 50$ & $02: 500$ & 02:49 & $02: 500$ & $02: 50$ & $02: 540$ & 402:590 & $02: 530$ & $02: 53$ & $302: 52$ & 202:57 & $02: 57$ & $702: 53$ & $302: 54$ & 03:03 & $02: 54$ \\
\hline \multirow{2}{*}{ After } & $5 \times 5 \mathrm{~m}$ & $17: 36$ & $16: 46$ & 20:03 & $20: 031$ & $13: 33$ & $19: 30$ & $25: 33$ & $20: 072$ & $20: 07$ & $713: 27$ & $16: 41$ & 16:41 & 114:17 & 714:17 & $21: 29$ & 18:01 \\
\hline & $10 \times 10 \mathrm{~m}$ & 03:50 & 04:40 & 03:32 & 03:330 & $03: 34$ & $403: 320$ & 203:500 & 03:260 & $03: 26$ & $603: 25$ & 04:13 & $03: 10$ & 003:05 & $505: 37 \mathrm{C}$ & 05:01 & $03: 52$ \\
\hline
\end{tabular}

\subsection{Terrain Level of Detail and Accuracy Comparison}

The research also carried out comparisons visibility analysis' accuracy ratio and limits by grids sizes. In case of Arcview, there were large numbers of differences on visibility based on grid sizes. For instance, there was even error producing Visibility Analysis on viewpoint 7 during the case study. There was a visible point in 1x1m grids; whereas, Arcview calculated the point was not visible within 10x10m grids. However, Arcview and VE3D produced similar results for $1 \mathrm{x} 1 \mathrm{~m}$ grids models. Therefore, it is reasonable to conclude that VE3D can be employed with $5 \times 5 \mathrm{~m}$ grids models for visibility analysis and evaluation with benefits of processing time and accuracy levels. Moreover, since there were negligible error on $1 \mathrm{x} 1 \mathrm{~m}$ and $5 \times 5 \mathrm{~m}$ grids models from Arcview, it is reasonable to compare Arcview with VE3D in $5 \times 5 \mathrm{~m}$ grids models.

Table 2. Visibility from Each Viewpoint (unit - per cent)

\begin{tabular}{|c|c|c|c|c|c|c|c|c|c|c|c|c|c|c|c|c|c|}
\hline \multirow{2}{*}{$\begin{array}{c}\text { View } \\
\text { Change }\end{array}$} & \multirow{2}{*}{ Software } & \multirow{2}{*}{ Grids } & \multicolumn{15}{|c|}{ Viewpoint } \\
\hline & & & 1 & 2 & 3 & 4 & 5 & 6 & 7 & 8 & 9 & 10 & 11 & 12 & 13 & 14 & 15 \\
\hline \multirow{5}{*}{ Before } & \multirow{3}{*}{ Arcview } & $1 \times 1 \mathrm{~m}$ & 59.6 & 64.4 & 64.4 & 66.4 & 67.6 & 42.2 & 43.0 & 40.2 & 33.8 & 29.5 & 15.1 & 16.2 & 17.2 & 17.9 & 18.6 \\
\hline & & $5 \times 5 \mathrm{~m}$ & 64.0 & 67.6 & 68.8 & 69.3 & 70.3 & 56.9 & 52.8 & 48.9 & 36.3 & 30.6 & 17.1 & 18.3 & 19.5 & 20.0 & 20.6 \\
\hline & & $10 \times 10 \mathrm{~m}$ & 67.5 & 70.5 & 72.4 & 71.8 & 72.7 & 50.0 & 11.5 & 45.5 & 40.9 & 36.4 & 19.5 & 21.1 & 21.8 & 22.2 & 23.2 \\
\hline & \multirow{2}{*}{ VE3D } & $5 \times 5 \mathrm{~m}$ & 56.6 & 60.6 & 63.1 & 63.3 & 63.9 & 52.5 & 48.4 & 41.1 & 35.3 & 34.1 & 24.2 & 26.1 & 27.5 & 28.6 & 28.7 \\
\hline & & $10 \times 10 \mathrm{~m}$ & 54.7 & 58.9 & 61.3 & 61.5 & 62.4 & 50.4 & 45.9 & 38.4 & 33.4 & 31.9 & 23.3 & 24.4 & 26.1 & 27.5 & 27.2 \\
\hline \multirow{2}{*}{ After } & \multirow{2}{*}{ Arcview } & $1 \times 1 \mathrm{~m}$ & \begin{tabular}{|l|}
8.9 \\
\end{tabular} & 8.8 & 7.3 & 9.8 & 11.9 & 9.9 & 9.4 & 9.7 & 9.1 & 8.7 & 11.7 & 12.1 & 12.4 & 12.8 & 12.9 \\
\hline & & $5 \times 5 \mathrm{~m}$ & 7.7 & 7.6 & 7.7 & 8.9 & 10.4 & 12.3 & 10.2 & 10.5 & 7.8 & 6.1 & 13.1 & 13.4 & 13.8 & 14.0 & 14.2 \\
\hline
\end{tabular}




\begin{tabular}{|c|c|c|c|c|c|c|c|c|c|c|c|c|c|c|c|c|}
\hline & $10 \times 10 \mathrm{~m}$ & 9.8 & 9.9 & 11.1 & 11.4 & 13.0 & 11.7 & 3.0 & 10.5 & 10.6 & 10.9 & 14.3 & 15.0 & 15.6 & \begin{tabular}{l|l}
6 & 15.4 \\
\end{tabular} & 15.2 \\
\hline \multirow{2}{*}{ VE3D } & $5 \times 5 \mathrm{~m}$ & 10.2 & 10.0 & 13.9 & 13.5 & 15.8 & 17.4 & 12.4 & 10.7 & 10.9 & 11.2 & 13.4 & 14.0 & 14.2 & \begin{tabular}{|l|l}
2 & 14.7 \\
\end{tabular} & 15.1 \\
\hline & $10 \times 10 \mathrm{~m}$ & 10.8 & 10.7 & 14.2 & 13.7 & 16.2 & 17.2 & 12.1 & 10.4 & 10.5 & 10.7 & 13.0 & 13.6 & 13.9 & $\begin{array}{ll}9 & 14.6\end{array}$ & 14.7 \\
\hline
\end{tabular}

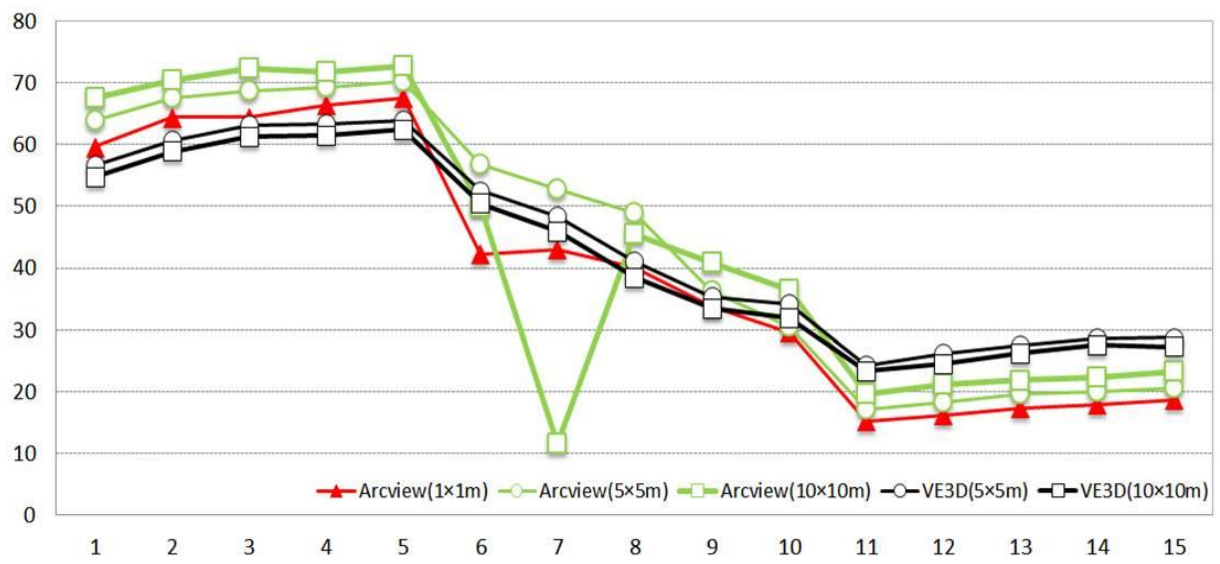

Figure 5. Relative Error Rates of Visible Areas (Before the Construction)

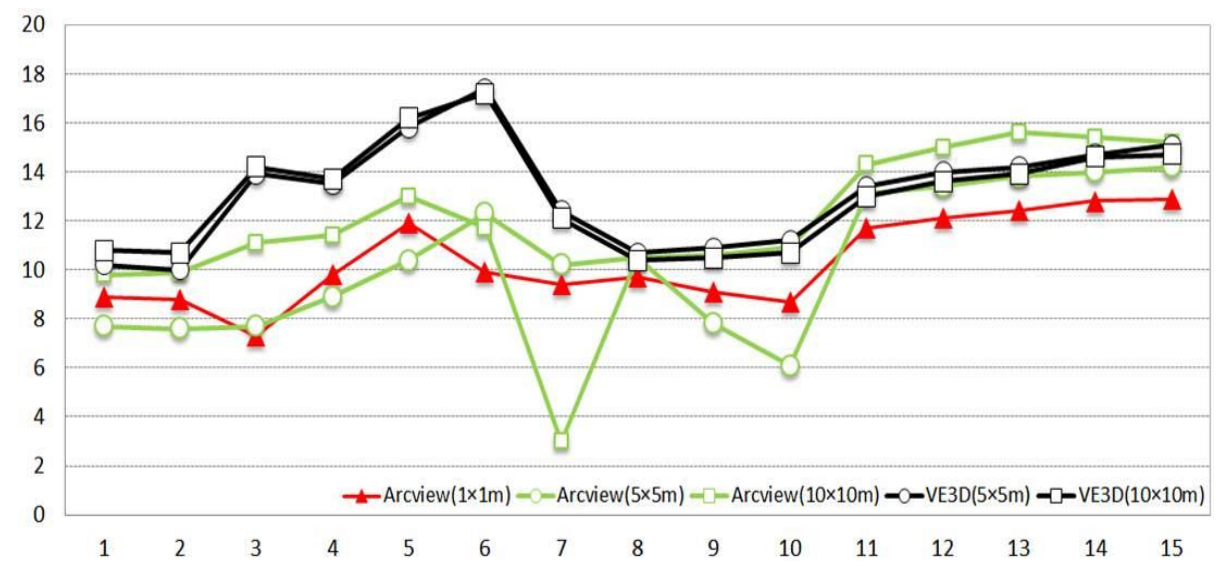

Figure 6. Relative Error Rates of Visible Areas (After the construction)

Nevertheless, it appears to be negligible differences on grid sizes for VE3D resulting visibility. Comparing differences of VE3D and Arcview on visible areas based on grids sizes, ArcView results have average 10-17\%; whereas, VE3D results revealed less than 5\% error rates. VE3D was found to be taken less impact from grid sizes; therefore, using 10x10m grids models, VE3D would be able to process Visibility Analysis efficiently without compromising accuracy.

\subsection{Visibility Analysis Comparison}

The research compared ArcView 3.3 and VE3D processes in $5 \times 5$ and 10x10m grids models. Then, visibility results in individual grids models were simulated with threedimensional models in order to compare human eye views to simulated results. There were a few areas where only ArcView analysis could identify within the before the construction analysis; however, there were large amount of areas where only VE3D analysis could identify in particular, around the concrete flyover and between tower blocks. This result explained that 
VE3D, NURBS/vector dataset based, is able to handle visibility analysis process in higher accuracy than ArcView, which is based on raster type methods.
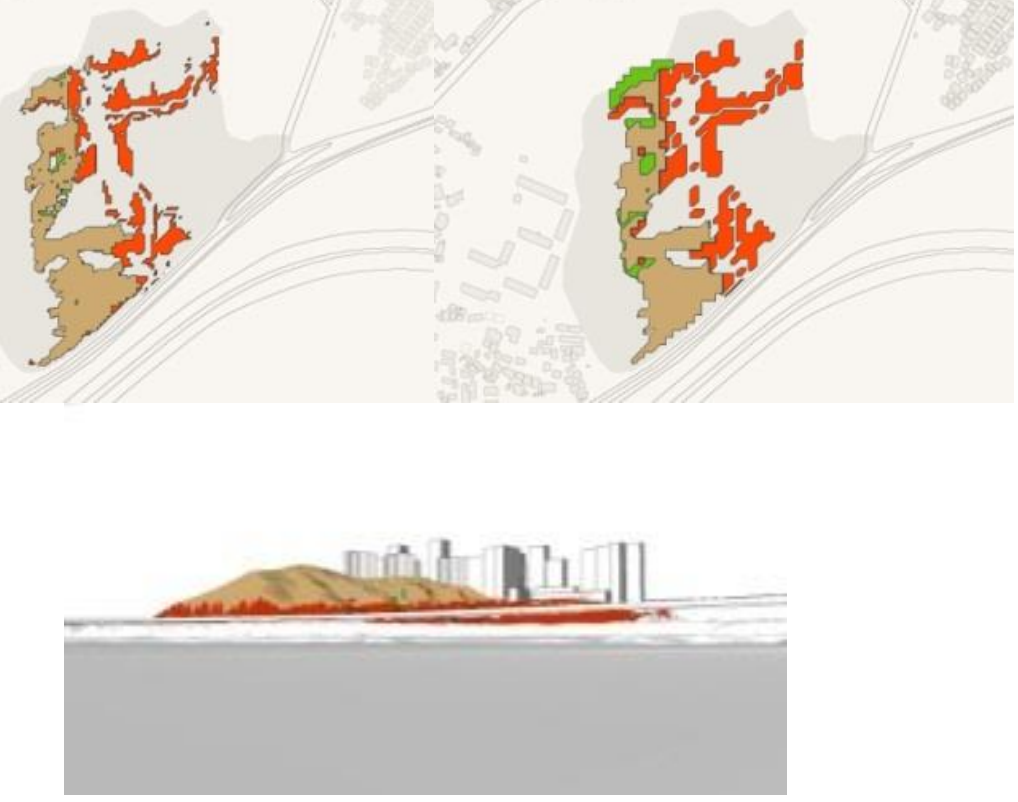

Figure 7. Visibility Analysis before the Construction of Tower Blocks (in Grids $5 \times 5,10 \times 10$, and Simulation)
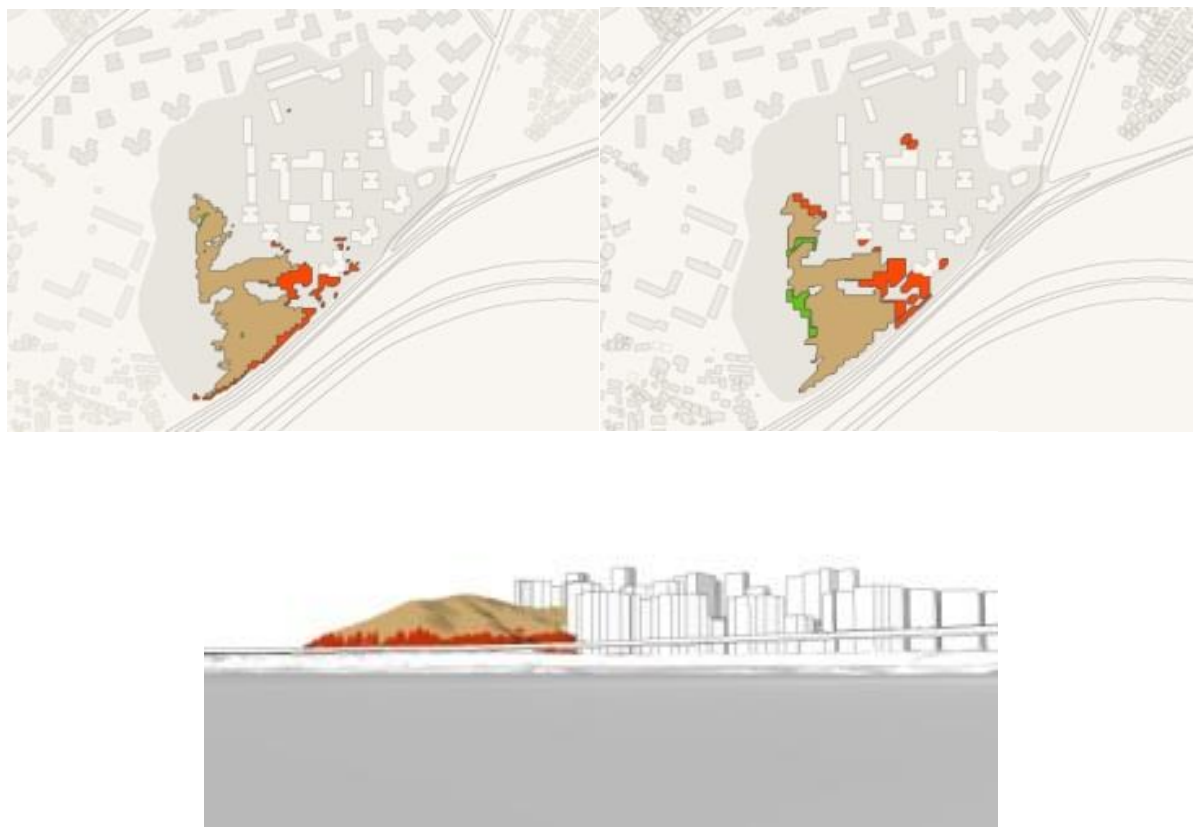

Figure 8. Visibility Analysis after the Construction of Tower Blocks (in Grids $5 \times 5,10 \times 10$, and Simulation) 


\subsection{Cumulative Visibility Analysis}

In order to compare Cumulative Visibility Analysis between ArcView 3.3 and VE3D generated, the research used $5 \times 5 \mathrm{~m}$ grids models and processed with same datasets. ArcView only used for Cumulative Visibility Analysis produced from rasterised individual analysis; whereas, VE3D was able to produce cumulative analysis based on distance-weighed and areaweighed values by $\mathrm{x}, \mathrm{y}, \mathrm{z}$ coordinates. Visual frequency from ArcView and VE3D was similar in the landscape before the construction; however, VE3D provided more intensive results as well as generated different results in northeast and north southern area of the site due to the existing flyover structures. Within the landscape after the construction, visual frequencies were very similar each other results from both ArcView and VE3D because of high-rise tower blocks were placed in the site.

The both distance and area weighed measures were produced by divisions from distance or double distance. Distance weighed method indicated that southern part of the site had excellent visibility and area weighed method results shows clear visibility in the southeast part of the site. This could be interpreted as different result to visual frequency. In short, if analysis weights more in viewpoints near the site rather than far off, visibility would be increased in the central or southern parts of the site. Therefore, it is reasonable to conclude that visibility is highly sensitive in central and eastern part of the site.

\section{Conclusions}

The study aimed to develop a Visibility Analysis programme based on NURBS in order to improve accuracy of the process and result. After case study of a residential development in Seoul, VE3D, which was developed for Visibility Analysis from vector-based datasets, was able to demonstrate and produce a result of high level of detail in visibility identification much better than using conventional ArcView 3.3 programme. Moreover, the VE3D was able to produce a Visibility Analysis in reflecting many complicated urban structures such as flyovers and high-rise tower blocks. In particular, carrying out a Visibility Analysis within 10x10m grids models, the VE3D can also perform the calculating process efficiently as well as maintaining high level of detail.

The research, therefore, suggests that the VE3D is able to contribute to in-depth and credible Visibility Analysis and this can be achieved by further research on application within various terrains and built up structures.

\section{Acknowledgement}

This work was supported by National Research Foundation of Korea - Grant funded by the Korean Government (Ministry of Science, ICT and Future Planning)-(NRF-2013K1A3A1A39075091).

\section{References}

[1] B.-J. Park, K. Furuya, T. Kasetani, N. Takayama, T. Kagawa and Y. Miyazaki, "Relationship between psychological responses and physical environments in forest settings", Landscape and Urban Planning, vol. 102, no. 1, (2011), pp. 24-32.

[2] J. M. Domingo-Santos, R. F. de Villarán, Í. Rapp-Arrarás, E. C.-P. de Provens, "The visual exposure in forest and rural landscapes: An algorithm and a GIS tool", Landscape and Urban Planning, vol. 101, no. 1, (2011), pp. 52-58.

[3] A. Jorgensen, "Beyond the view: Future directions in landscape aesthetics research", Landscape and Urban Planning, vol. 100, no. 1, (2011), pp. 353-355.

[4] J.B. Kirkpatrick, G.D. Daniels, A. Davison, "Temporal and spatial variation in garden and street trees in six eastern Australian cities", Landscape and Urban Planning, vol. 1021, no. 3, (2011), pp. 244-252. 
[5] G. Domon, "Landscape as resource: Consequences, challenges and opportunities for rural development", Landscape and Urban Planning, vol. 100, no. 1, (2011), pp. 338-340.

[6] Z. Zheng and Z. Bohong, "Study on Spatial Structure of Yangtze River Delta Urban Agglomeration and Its Effects on Urban and Rural Regions", Landscape and Urban Planning and Development-ASCE, vol. 138, no. 1, (2012), pp. 78-89.

[7] D. Lee and H. Choe, "Estimating the Impacts of Urban Expansion on Landscape Ecology: Forestland Perspective in the Greater Seoul Metropolitan Area", Journal of Urban Planning and development"-ASCE, vol. 137, no. 4, (2011), pp. 425-437.

[8] P. C. Anastasopoulos, M. B. Islam, D. Perperidou, and M. G. Karlaftis, "Hazard-Based Analysis of Travel Distance in Urban Environments: Longitudinal Data Approach", Journal of Urban Planning and development"-ASCE, vol. 138, no. 1, (2012), pp. 53-61.

[9] A. T. Oakil, D. Ettema, T. Arentze and H. Timmermans, "Longitudinal Model of Longer-Term Mobility Decisions: Framework and First Empirical Tests", Journal of Urban Planning and development"-ASCE, vol. 137, no. 3, (2011), pp. 220-229.

[10] E. C. Hui, I. M. Ng and K. Lo, "Analysis of the Viability of an Urban Renewal Project under a Risk-Based Option Pricing Framework", Journal of Urban Planning and development"-ASCE, vol. 137, no. 2, (2011), pp. 101-111.

[11] K. J. An, H. K. Kang, G. Marreiros and H. Ko, "Utilisation of Photo-montages for UK planning", Information Journal, 15:11(2012B), 4785-4796.

\section{Authors}
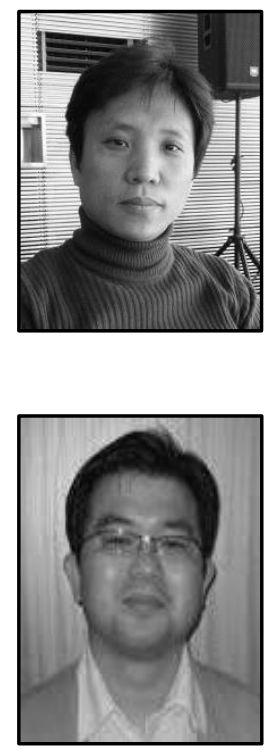

Choong Sik Kim, He received the Bachelor's degree, Master's degree and $\mathrm{Ph}$. D. in Landscape Architecture from The University of Seoul, Seoul, Korea, in 1995, 1998 and 2004 respectively. He has previously worked at KangnuengWonju National University, Kangwondo as an associate professor from 2007 to 2013. He is an assistant professor of the Department of Traditional Landscape Architecture in Korea National University of Cultural Heritage. His research interests lie in the area of quantitative landscape analysis especially in cumulative visibility.

Hoon Ko, He got the B.S. degree in computer science from Howon University, Kunsan-City, S. Korea, in 1998, and M.S. degree in computer science from Soongsil University, Seoul, S. Korea in 2000 and Ph D. degree in 2004. He had joined in Daejin University as a visiting professor from 2002 to 2006. He had worked at Information \& Communications University (ICU), Korea Advanced Institute of Science and Technology (KAIST) in 2007. Next, he had worked at GECAD, ISEP, IPP in Porto, Portugal as a Doctor Researcher from 2008 to 2013. Now he is a research professor at the department of informatics, University of J. E. Purkinje since 2013. He is interested to Urban Computing Security, Ubiquitous Computing Security, AmI Security, ContextAware Security, MSEC(Multicast Security), RFID Security, Home Network Security, etc.

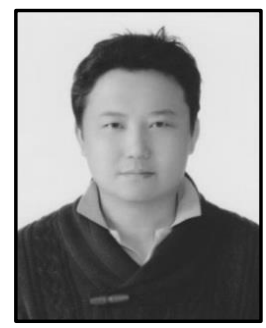

KyungJin An, Kyung in received BSc in landscape architecture from University of Seoul, South Korea. In 2002, he completed Master of Landscape Architecture in Newcastle University in the UK with Golden Jubilee Award. During the period between 2002 and 2012, he read PhD in School of Architecture, Planning and Landscape with scholarships from Newcastle University. $\mathrm{He}$ is particularly interested in computer visualisation and its implementation into design industry. He has published a number of papers in the field and continues to research on the same subject. Now, he is an assistant professor at Konkuk University since 2013. 
International Journal of Smart Home

Vol. 9, No. 1 (2015) 